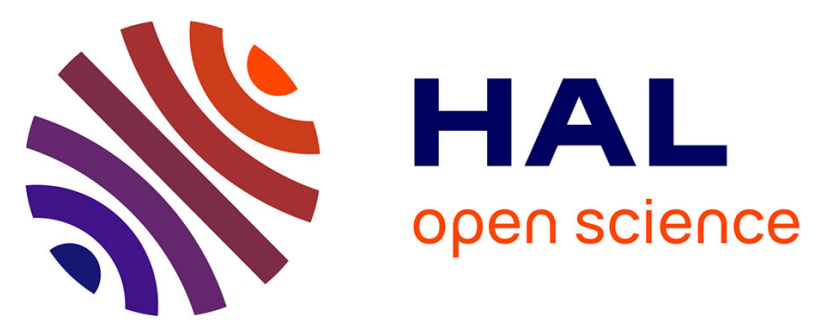

\title{
A Class of Nonlinear Unknown Input Observer for Fault Diagnosis: Application to Fault Tolerant Control of an Autonomous Spacecraft
}

Robert Fonod, David Henry, Catherine Charbonnel, Eric Bornschlegl

\section{- To cite this version:}

Robert Fonod, David Henry, Catherine Charbonnel, Eric Bornschlegl. A Class of Nonlinear Unknown Input Observer for Fault Diagnosis: Application to Fault Tolerant Control of an Autonomous Spacecraft. 10th UKACC International Conference on Control, United Kingdom Automatic Control Council (UKACC), Jul 2014, Loughborough, United Kingdom. pp.19-24, 10.1109/CONTROL.2014.6915108 . hal-00989423v3

\section{HAL Id: hal-00989423 \\ https://inria.hal.science/hal-00989423v3}

Submitted on 16 Jun 2016

HAL is a multi-disciplinary open access archive for the deposit and dissemination of scientific research documents, whether they are published or not. The documents may come from teaching and research institutions in France or abroad, or from public or private research centers.
L'archive ouverte pluridisciplinaire $\mathbf{H A L}$, est destinée au dépôt et à la diffusion de documents scientifiques de niveau recherche, publiés ou non, émanant des établissements d'enseignement et de recherche français ou étrangers, des laboratoires publics ou privés. 


\title{
A Class of Nonlinear Unknown Input Observer for Fault Diagnosis: Application to Fault Tolerant Control of an Autonomous Spacecraft
}

\author{
Robert Fonod*, David Henry*, Catherine Charbonnel ${ }^{\dagger}$ and Eric Bornschlegl ${ }^{\ddagger}$ \\ *Université de Bordeaux, IMS UMR CNRS 5218, Automatic Control Group, Talence, France \\ ${ }^{\dagger}$ Thales Alenia Space, RT/SO Research Department, Cannes la Bocca, France \\ ${ }^{\ddagger}$ European Space Agency, ESTEC, TEC-ECN, Noordwijk, Netherlands \\ ${ }^{*}$ Contact: \{robert.fonod, david.henry\}@ims-bordeaux.fr
}

\begin{abstract}
In this paper, the problem of Nonlinear Unknown Input Observer (NUIO) based Fault Detection and Isolation (FDI) scheme design for a class of nonlinear Lipschitz systems is studied. The proposed FDI method is applied to detect, isolate and accommodate thruster faults of an autonomous spacecraft involved in the rendezvous phase of the Mars Sample Return (MSR) mission. Considered fault scenarios represent fully closed thruster and thruster efficiency loss. The FDI scheme consists of a bank of NUIOs with adjustable error dynamics, a robust fault detector that is based on judiciously chosen frame and an isolation logic. The bank of observers is in charge of confining the fault to a subset of possible faults and the isolation logic makes the final decision about the faulty thruster index. Finally, a thruster fault is accommodated by re-allocating the desired forces and torques among the remaining healthy thrusters and closing the associated thruster valve. Monte Carlo results from "high-fidelity" MSR industrial simulator demonstrate that the proposed fault tolerant strategy is able to accommodate thruster faults that may have effect on the final rendezvous criteria.
\end{abstract}

\section{INTRODUCTION}

In the recent decades, due to the increased complexity, as well as, the need for reliability, safety, and efficient operation, a great deal of attention has been paid to the subject of Fault/Failure Detection Isolation and Recovery (FDIR) in space systems, see for instance [1], [2]. Literature reports that conventional FDIR approaches suffer from significant shortcomings, like increased mass and system complexity, often missing on-board isolation of the faults, ground intervention is not always possible due to large communication delays or visibility issues, and knowledge about the operational capabilities of the system is not present on-board. Existing FDIR techniques used in space systems are industrially well mastered but may be not sufficient in some cases, e.g. when a dynamic deviation in critical/proximity space operation could possibly lead to mission loss. This fact motivates the European Space Agency (ESA) to lead studies for the development of fully autonomous on-board solutions that shall cope with all the possible faults, that may occur and endanger mission.

Advanced Fault Detection and Isolation (FDI) approaches should be specifically developed to safely conjugate the necessary robustness/stability of the spacecraft control, trajectory dynamics and the vehicle nominal performance. In order to ensure the normal operation, real-time fault detection and isolation is necessary to provide information for the spacecraft to accommodate the fault in time. The presented work is a result of a research collaboration between ESA, Thales Alenia Space and IMS Laboratory with the aim of promoting
Fault-Tolerant Control (FTC) strategies to advance spacecraft autonomy. The application concerns the rendezvous phase of the Mars Sample Return (MSR) mission. The goal of the mission is to return samples from Mars to the Earth for analysis (see [3] for more details about this mission). It is obvious, that the rendezvous phase can be in danger, if thruster fault occurs. The Guidance, Navigation and Control (GNC) system may not compensate, e.g. J2 disturbances and/or may lose attitude and/or position of the sample container (target). The problem becomes highly critical during the last 20 meters of the rendezvous phase. During this phase, the chaser spacecraft must be correctly positioned in the approach corridor in order to successfully capture the target, as well, as the chaser's attitude need to be maintained in the sensors' field of view.

Growing interest for potential applications of model-based FDI algorithms in spacecraft systems is demonstrated by recent publications, see e.g. [4]-[7]. In terms of FTC techniques, the interested reader may refer to the excellent bibliographical review of Zhang and Jiang [8], who explain the existing approaches on this topic. A special class of observer based FDI approaches is the so-called Unknown Input Observer (UIO). Under certain conditions, UIO is able to estimate the state, when exogenous unknown inputs are present in the system. This property may be sometimes very useful for FDI scheme design. Linear UIO algorithms [9], [10] are extended for various classes of nonlinear systems in [11]-[13]. In [12], $H_{\infty}$ optimization based Nonlinear Unknown Input Observer (NUIO) design is presented. The observer is called dynamic UIO which offers an extra degree of design freedom, but increases the system's order. In [13], a NUIO is presented for a class of nonlinear systems. The design procedure is based on Linear Matrix Inequalities (LMIs).

In this paper, we consider a NUIO based FDI scheme design problem for a class of nonlinear Lipschitz systems. We extend the results presented in [14] by constraining the observer error dynamics in a prescribed LMI region. The observer synthesis is achieved by solving a LMI feasibility problem together with a pole assignment in LMI regions. Thus, a bank of NUIOs can be designed such that the error dynamics of each NUIO is easily tunable. This bank is used to identify a subset of thrusters that are most likely faulty. Blocked-closed and loss of efficiency thruster faults are considered. Once a fault is isolated, the remaining $N-1$ healthy thrusters are used to control the spacecraft. This fault accommodation strategy is achieved by control re-allocation technique. By this, the nominal (in-placed and certified) control laws remain unchanged which is a prior condition from an industrial perspective. 


\section{PRELIMINARIES: LMI REGIONS}

Chilali and Gahinet [15] showed, that a convex set that represents the desired constraints on the eigenvalues of a real matrix can be expressed as LMIs. We recall here the definition of an LMI region and the pole placement LMI constraints.

Definition 1 (LMI region [15]): A subset $\mathcal{D}$ of the complex plane is called an LMI region if there exist two symmetric matrices $\boldsymbol{\alpha}=\left[\alpha_{k l}\right] \in \mathbb{R}^{p \times p}$ and $\boldsymbol{\beta}=\left[\beta_{k l}\right] \in \mathbb{R}^{p \times p}$, such that

$$
\mathcal{D}=\left\{z \in \mathcal{C}: \boldsymbol{f}_{\mathcal{D}}(z)=\boldsymbol{\alpha}+\boldsymbol{\beta} z+\boldsymbol{\beta}^{T} \bar{z}<0\right\}
$$

where $\boldsymbol{f}_{\mathcal{D}}(z)$ is called the characteristic function.

Theorem 1: Eigenvalues of a real matrix $\boldsymbol{X}$ lie in $\mathcal{D}$, if and only if there exists a symmetric positive definite matrix $\boldsymbol{P}>0$, such that

$$
\boldsymbol{\alpha} \otimes \boldsymbol{P}+\boldsymbol{\beta} \otimes(\boldsymbol{A P})+\boldsymbol{\beta}^{T} \otimes(\boldsymbol{A P})^{T}<0
$$

where $\otimes$ stands for the Kronecker product of two matrices. Examples of LMI regions from definition 1 are:

- Left-half plane delimited by a vertical line $-a, a>0$

$$
\boldsymbol{f}_{\mathcal{D}}(z)=z+\bar{z}+2 a
$$

- Disk with center at $(-q, 0)$ and radius $r$ with $q>0$

$$
\boldsymbol{f}_{\mathcal{D}}(z)=\left(\begin{array}{cc}
-r & q+z \\
q+\bar{z} & -r
\end{array}\right)
$$

- Conic region with center at the origin and with inner angle $0<\theta<\pi / 2$ pointing left

$$
\boldsymbol{f}_{\mathcal{D}}(z)=\left(\begin{array}{ll}
\sin \theta(z+\bar{z}) & \cos \theta(z-\bar{z}) \\
\cos \theta(z-\bar{z}) & \sin \theta(z+\bar{z})
\end{array}\right)
$$

LMIs can be easily obtained from (3)-(5) using Theorem 1

\section{NONLINEAR UNKNOWN INPUT OBSERVER}

\section{A. Problem statement}

Let us consider the following nonlinear system given by

$$
\begin{aligned}
& \dot{\boldsymbol{x}}(t)=\boldsymbol{A} \boldsymbol{x}(t)+\boldsymbol{B} \boldsymbol{u}(t)+\boldsymbol{f}(\boldsymbol{x}(t))+\boldsymbol{E d}(t) \\
& \boldsymbol{y}(t)=\boldsymbol{C x}(t)
\end{aligned}
$$

where $\boldsymbol{x} \in \mathbb{R}^{n}$ stands for the state vector, $\boldsymbol{y} \in \mathbb{R}^{n_{m}}$ is the output, $\boldsymbol{u} \in \mathbb{R}^{n_{r}}$ is the input, and $\boldsymbol{d} \in \mathbb{R}^{n_{q}}$ is the unknown input (disturbance or fault) vector. $\boldsymbol{A}, \boldsymbol{B}, \boldsymbol{C}$, and $\boldsymbol{E}$ are known matrices of appropriate dimensions. Without loss of generality, it is assumed that $\boldsymbol{E}$ is of full column rank.

The known function $\boldsymbol{f}(\boldsymbol{x}) \in \mathbb{R}^{n}$ contains the nonlinearities of the system. Assume that $\boldsymbol{f}(\boldsymbol{x})$ is globally Lipschitz or at least locally Lipschitz in a region $\mathcal{S}$, i.e. it satisfies:

$$
\begin{gathered}
\left\|\boldsymbol{f}\left(\boldsymbol{x}_{1}\right)-\boldsymbol{f}\left(\boldsymbol{x}_{2}\right)\right\| \leq \kappa\left\|\boldsymbol{x}_{1}-\boldsymbol{x}_{2}\right\| \\
\begin{cases}\forall \boldsymbol{x}_{1}, \boldsymbol{x}_{2} \in \mathbb{R}^{n} & \text { globally Lipschitz } \\
\forall \boldsymbol{x}_{1}, \boldsymbol{x}_{2} \in \mathcal{S} & \text { locally Lipschitz }\end{cases}
\end{gathered}
$$

where $\kappa>0$ stands for the Lipschitz constant and $\|\cdot\|$ is the Euclidian norm. Many nonlinearities satisfy (8) at least locally.

The goal which is pursued is to design a NUIO of the following structure [13]:

$$
\begin{aligned}
& \dot{\boldsymbol{z}}(t)=\boldsymbol{N} \boldsymbol{z}(t)+\boldsymbol{G u}(t)+\boldsymbol{L} \boldsymbol{y}(t)+\boldsymbol{M} \boldsymbol{f}(\hat{\boldsymbol{x}}(t)) \\
& \hat{\boldsymbol{x}}(t)=\boldsymbol{z}(t)-\boldsymbol{H} \boldsymbol{y}(t)
\end{aligned}
$$

where $\hat{\boldsymbol{x}} \in \mathbb{R}^{n}$ is an estimate of $\boldsymbol{x}, \boldsymbol{z} \in \mathbb{R}^{n}$ is an auxiliary signal and the matrices $N, G, L, M$ are designed as

$$
\begin{aligned}
\boldsymbol{N} & =\boldsymbol{M A}-\boldsymbol{K} \boldsymbol{C}, \quad \boldsymbol{G}=\boldsymbol{M B} \\
\boldsymbol{L} & =\boldsymbol{K}(\boldsymbol{I}+\boldsymbol{C H})-\boldsymbol{M A H} \\
\boldsymbol{M} & =\boldsymbol{I}+\boldsymbol{H} \boldsymbol{C}
\end{aligned}
$$

and $\boldsymbol{K}$ and $\boldsymbol{H}$ are designed subsequently.

Define the state estimation error as

$$
\boldsymbol{e}(t)=\hat{\boldsymbol{x}}(t)-\boldsymbol{x}(t)=\boldsymbol{z}(t)-\boldsymbol{H} \boldsymbol{y}(t)-\boldsymbol{x}(t)
$$

Taking the time derivative of the estimation error yields

$$
\begin{aligned}
\dot{\boldsymbol{e}}(t)= & \boldsymbol{N} \boldsymbol{e}(t)+(\boldsymbol{N} \boldsymbol{M}+\boldsymbol{L} \boldsymbol{C}-\boldsymbol{M} \boldsymbol{A}) \boldsymbol{x}(t)-\boldsymbol{M} \boldsymbol{E} \boldsymbol{d}(t) \\
& +(\boldsymbol{G}-\boldsymbol{M} \boldsymbol{B}) \boldsymbol{u}(t)+\boldsymbol{M}(\boldsymbol{f}(\hat{\boldsymbol{x}}(t))-\boldsymbol{f}(\boldsymbol{x}(t))
\end{aligned}
$$

Definition 2 (Adjustable NUIO): In this paper, an observer of the form (9)-(10) is referred to as an adjustable NUIO for the system (6)-(7) if the estimation error tends asymptotically to zero despite the presence of an unknown input $\boldsymbol{d}(t) \neq \mathbf{0}$ and if all eigenvalues of the observer dynamics matrix $N$ lies in a prescribed region $\mathcal{D}$ of the complex left-half plane.

This definition accommodates the observer given in [13], such that the state estimation error has an adjustable error dynamics. The sufficient condition under which the observer given by (9)10] is an adjustable NUIO is given in Theorem 2 .

Theorem 2 (Sufficient condition): Let $\mathcal{D}$ be an LMI region contained in the complex left-half plane and with (1). If there exist two matrices $\boldsymbol{H}$ and $\boldsymbol{K}$ and a positive definite matrix $\boldsymbol{P}=\boldsymbol{P}^{T}>0$ such that

$$
\begin{aligned}
& \boldsymbol{H C} \boldsymbol{E}=-\boldsymbol{E} \\
& \boldsymbol{N}^{T} \boldsymbol{P}+\boldsymbol{P} \boldsymbol{N}+\kappa \boldsymbol{P} \boldsymbol{M} \boldsymbol{M}^{T} \boldsymbol{P}+\kappa \boldsymbol{I}<0 \\
& \boldsymbol{\alpha} \otimes \boldsymbol{P}+\boldsymbol{\beta} \otimes(\boldsymbol{N} \boldsymbol{P})+\boldsymbol{\beta}^{T} \otimes(\boldsymbol{N} \boldsymbol{P})^{T}<0
\end{aligned}
$$

then the adjustable NUIO given by 9 - 10 can make $e(t)$ tend to zero asymptotically for any $\boldsymbol{e}(0)$ and all eigenvalues of the observer dynamics matrix $N$ will belong to $\mathcal{D}$.

Proof: Using (11)-(13) equalities $N \boldsymbol{M}+\boldsymbol{L} \boldsymbol{C}-\boldsymbol{M} \boldsymbol{A}=\mathbf{0}$ and $\boldsymbol{G}-\boldsymbol{M B}=\mathbf{0}$ are satisfied, and if $\boldsymbol{H}$ is chosen such that (15) holds, then the condition (15) can be rewritten as

$$
\boldsymbol{M E}=(\boldsymbol{I}+\boldsymbol{H C}) \boldsymbol{E}=\mathbf{0}
$$

and therefore the error dynamics is be governed by

$$
\dot{\boldsymbol{e}}(t)=\boldsymbol{N} \boldsymbol{e}(t)+\boldsymbol{M}(\boldsymbol{f}(\hat{\boldsymbol{x}}(t))-\boldsymbol{f}(\boldsymbol{x}(t)))
$$

Consider a quadratic Lyapunov function $V(t)=\boldsymbol{e}(t)^{T} \boldsymbol{P} \boldsymbol{e}(t)$, then it follows from (8) and (19) that

$$
\begin{aligned}
\dot{\boldsymbol{V}} & =\boldsymbol{e}^{T}\left(\boldsymbol{N}^{T} \boldsymbol{P}+\boldsymbol{P} \boldsymbol{N}\right) \boldsymbol{e}+2 \boldsymbol{e}^{T} \boldsymbol{P} \boldsymbol{M}(\boldsymbol{f}(\hat{\boldsymbol{x}})-\boldsymbol{f}(\boldsymbol{x})) \\
& \leq \boldsymbol{e}^{T}\left(\boldsymbol{N}^{T} \boldsymbol{P}+\boldsymbol{P} \boldsymbol{N}\right) \boldsymbol{e}+2\left\|\boldsymbol{e}^{T} \boldsymbol{P} \boldsymbol{M}\right\|\|\boldsymbol{f}(\hat{\boldsymbol{x}})-\boldsymbol{f}(\boldsymbol{x})\| \\
& \leq \boldsymbol{e}^{T}\left(\boldsymbol{N}^{T} \boldsymbol{P}+\boldsymbol{P} \boldsymbol{N}\right) \boldsymbol{e}+2 \kappa\left\|\boldsymbol{e}^{T} \boldsymbol{P} \boldsymbol{M}\right\|\|\boldsymbol{e}\| \\
& \leq \boldsymbol{e}^{T}\left(\boldsymbol{N}^{T} \boldsymbol{P}+\boldsymbol{P} \boldsymbol{N}\right) \boldsymbol{e}+\kappa\left(\left\|\boldsymbol{e}^{T} \boldsymbol{P} \boldsymbol{M}\right\|^{2}+\|\boldsymbol{e}\|^{2}\right) \\
& =\boldsymbol{e}^{T}\left(\boldsymbol{N}^{T} \boldsymbol{P}+\boldsymbol{P} \boldsymbol{N}+\kappa \boldsymbol{P} \boldsymbol{M} \boldsymbol{M}^{T} \boldsymbol{P}+\kappa \boldsymbol{I}\right) \boldsymbol{e}
\end{aligned}
$$

Obviously, $\dot{\boldsymbol{V}}<0$ holds if (16) is satisfied, thus $\lim _{t \rightarrow \infty} \boldsymbol{e}(t)=\mathbf{0}$ for any $e(0)$. Moreover, if (16)-(17) are satisfied at the same time, Theorem 1 implies that all eigenvalues of $N$ are in $\mathcal{D}$.

Remark 1: The LMI (17) itself does not impose stability of (19), even if all eigenvalues of $N$ lies in a stable region $\mathcal{D}$. 
The necessary condition for $\boldsymbol{H C E}=-\boldsymbol{E}$ to have a solution is that $\boldsymbol{C E}$ is of full column rank, i.e. $\operatorname{rank}(\boldsymbol{C E})=n_{q}$, and the solution is given in a generalized form by

$$
\boldsymbol{H}=\boldsymbol{U}+\boldsymbol{Y} \boldsymbol{V}
$$

where $\boldsymbol{Y}$ can be chosen arbitrarily, $\boldsymbol{U}$ and $\boldsymbol{V}$ are given by

$$
\boldsymbol{U}=-\boldsymbol{E}(\boldsymbol{C E})^{+}, \quad \boldsymbol{V}=\boldsymbol{I}-(\boldsymbol{C E})(\boldsymbol{C E})^{+}
$$

and $(\boldsymbol{C E})^{+}$denotes the generalized pseudo-inverse of the matrix $\boldsymbol{C E}$ given by $(\boldsymbol{C E})^{+}=\left((\boldsymbol{C E})^{T}(\boldsymbol{C E})\right)^{-1}(\boldsymbol{C E})^{T}$.

It is clear that there is no systematic way to obtain the adjustable NUIO parameters directly from Theorem 2. This motivates us to reformulate (16)-(17) as LMIs.

\section{B. LMI formulation}

For sake of simplicity, let $\mathcal{D}$ be a LMI region defining a disk with a center $(-q, 0)$ and a radius $r>0$. Since $\boldsymbol{H}$ can be computed using (20), the only unknown parameters in (11)(13) are $\boldsymbol{K}$ and $\boldsymbol{Y}$. The following theorem shows that the sufficient condition of existence given by Theorem 2 can be reformulated as LMIs to design the NUIO parameters.

Proposition 1 (LMI based design): Assume that $\boldsymbol{C E}$ is of full column rank and that the following LMIs

$$
\begin{gathered}
{\left[\begin{array}{cc}
\boldsymbol{Q}_{1} & \boldsymbol{Q}_{2} \\
* & -\boldsymbol{I}
\end{array}\right]<0} \\
{\left[\begin{array}{cc}
-r \boldsymbol{P} & q \boldsymbol{P}+\boldsymbol{Q}_{3} \\
* & -r \boldsymbol{P}
\end{array}\right]<0}
\end{gathered}
$$

where $*$ denotes the symmetric item in a symmetric matrix, and $\boldsymbol{Q}_{1}, \boldsymbol{Q}_{2}$ and $\boldsymbol{Q}_{3}$ are defined as

$$
\begin{aligned}
\boldsymbol{Q}_{1}= & ((\boldsymbol{I}+\boldsymbol{U} \boldsymbol{C}) \boldsymbol{A})^{T} \boldsymbol{P}+\boldsymbol{P}(\boldsymbol{I}+\boldsymbol{U} \boldsymbol{C}) \boldsymbol{A}-\boldsymbol{C}^{T} \overline{\boldsymbol{K}}^{T} \\
& -\overline{\boldsymbol{K}} \boldsymbol{C}+(\boldsymbol{V} \boldsymbol{C} \boldsymbol{A})^{T} \overline{\boldsymbol{Y}}^{T}+\overline{\boldsymbol{Y}}(\boldsymbol{V} \boldsymbol{C} \boldsymbol{A})+\kappa \boldsymbol{I} \\
\boldsymbol{Q}_{2}= & \sqrt{\kappa}[\boldsymbol{P}(\boldsymbol{I}+\boldsymbol{U} \boldsymbol{C})+\overline{\boldsymbol{Y}}(\boldsymbol{V} \boldsymbol{C})] \\
\boldsymbol{Q}_{3}= & \boldsymbol{A}^{T} \boldsymbol{P}+(\boldsymbol{U} \boldsymbol{C} \boldsymbol{A})^{T} \boldsymbol{P}+(\boldsymbol{V} \boldsymbol{C} \boldsymbol{A})^{T} \overline{\boldsymbol{Y}}^{T}-\boldsymbol{C}^{T} \overline{\boldsymbol{K}}^{T}
\end{aligned}
$$

have feasible solutions for $\overline{\boldsymbol{Y}}, \overline{\boldsymbol{K}}$ and $\boldsymbol{P}=\boldsymbol{P}^{T}>0$. Moreover, if (22) and (23) are fulfilled simultaneously with the same $\overline{\boldsymbol{Y}}$, $\overline{\boldsymbol{K}}$ and $\boldsymbol{P}$, then the adjustable NUIO given by (9)-(10) can be designed with $\boldsymbol{Y}=\boldsymbol{P}^{-1} \overline{\boldsymbol{Y}}$, and $\boldsymbol{K}=\boldsymbol{P}^{-1} \overline{\boldsymbol{K}}$ making all eigenvalues of $\boldsymbol{N}$ lying inside a disk centered at $(-q, 0)$ with radius $r$ and the estimation error $\boldsymbol{e}(t)=\hat{\boldsymbol{x}}(t)-\boldsymbol{x}(t)$ tending to zero asymptotically for any initial value of $\boldsymbol{e}(0)$.

Proof: It is straightforward to show that (22) is equivalent to (16) if we let $\boldsymbol{H}=\boldsymbol{U}+\boldsymbol{Y} \boldsymbol{V}$ and $\boldsymbol{M}=\boldsymbol{I}+\boldsymbol{H C}$, and if we substitute $\boldsymbol{N}$ given by (11) into 16 and use Schur's complement and assignment $\boldsymbol{Y}=\boldsymbol{P}^{-1} \boldsymbol{Y}$ and $\boldsymbol{K}=\boldsymbol{P}^{-1} \overline{\boldsymbol{K}}$. Thus, asymptotical stability yields. The LMI region with characteristic function (4) gives the following LMI (see [15]):

$$
\left[\begin{array}{cc}
-r \boldsymbol{P} & q \boldsymbol{P}+\boldsymbol{X} \boldsymbol{P} \\
* & -r \boldsymbol{P}
\end{array}\right]<0, \quad \boldsymbol{P}=\boldsymbol{P}^{T}>0
$$

Using the fact that the eigenvalues of any square matrix $\boldsymbol{X}$ are equal to the eigenvalues of its transpose $\boldsymbol{X}^{T}$, then by the following notation $\boldsymbol{X}^{T}=\boldsymbol{N}=\boldsymbol{A}+\boldsymbol{U} \boldsymbol{C A}+\boldsymbol{Y} \boldsymbol{V} \boldsymbol{C A}-\boldsymbol{K} \boldsymbol{C}$, it follows that 24) implies (23). All eigenvalues of $N$ will therefore lie inside a disk of radius $r$ and center $(-q, 0)$. Note that $\boldsymbol{H}=\boldsymbol{U}+\boldsymbol{Y} \boldsymbol{V}$ implies $\boldsymbol{H} \boldsymbol{C E}=-\boldsymbol{E}$, i.e. all conditions required by Theorem 2 are met and the theorem is proved.

\section{Solution for Thruster Fault Detection AND ISOLATION PROBLEM}

In the following, it is shown how a bank of NUIOs can be used to isolate actuator faults in the chaser spacecraft of the MSR mission. The chaser is equipped with a chemical propulsion system composed of $N=12$ thrusters. The considered thruster configuration in this paper is not a baseline MSR configuration but a special one designed by Thales Alenia Space to study active FTC strategies. The thrusters are physically organised in four clusters and are in charge of producing force $\boldsymbol{F} \in \mathbb{R}^{3}$ and torque $\boldsymbol{T} \in \mathbb{R}^{3}$ vectors expressed in the chaser body-fixed reference frame $\mathcal{F}_{b}=\left\{O_{b} ; \hat{\boldsymbol{x}}_{b}, \hat{\boldsymbol{y}}_{b}, \hat{\boldsymbol{z}}_{b}\right\}$. Let $\mathcal{S}_{\text {all }}=\{1,2, \ldots N\}$ denote the set of all thruster indices. Thrusters have fixed directions $\boldsymbol{d}_{i} \in \mathbb{R}^{3}, \forall i \in \mathcal{S}_{\text {all }}$ and each one is able to produce a maximum thrust of $F_{N}=22 \mathrm{~N}$. The Chemical Propulsion Drive Electronics (CPDE), that drives the thrusting actuators, is initiating the opening of the thruster valve for the commanded duration $0 \leq u_{i}(t) \leq 1, \forall i \in \mathcal{S}_{\text {all }}$. The propulsion system is obviously a source of uncertainty in the system. The irrational transfer $H(s)=e^{-\tau(t) s}$ aims to model the effect of the unknown time-varying delays $\tau(t) \geq 0$ induced by the CPDE and the uncertainties on the thruster rise times. Let $u_{i}(t-\tau(t))$ be the commanded open rate of the $i^{t h}$ thruster delayed by $\tau(t)$, then the net forces and torques generated by thrusters are

$$
\begin{aligned}
& \qquad \boldsymbol{F}(t)=\boldsymbol{B}_{F} \boldsymbol{u}(t-\tau(t)), \quad \boldsymbol{T}(t)=\boldsymbol{B}_{T} \boldsymbol{u}(t-\tau(t)) \\
& \text { where } \boldsymbol{u}(t)=\left[\begin{array}{lll}
u_{1}(t) & \ldots & u_{12}(t)
\end{array}\right]^{T}, \text { and } \\
& \qquad \boldsymbol{B}_{F}=\left[\begin{array}{llll}
\boldsymbol{b}_{F_{1}} & \ldots & \boldsymbol{b}_{F_{12}}
\end{array}\right], \quad \boldsymbol{B}_{T}=\left[\begin{array}{lll}
\boldsymbol{b}_{T_{1}} & \ldots & \boldsymbol{b}_{T_{12}}
\end{array}\right]
\end{aligned}
$$

are the thruster sensitivity (configuration) matrices with

$$
\boldsymbol{b}_{F_{i}}=-\boldsymbol{d}_{i} F_{N}, \quad \boldsymbol{b}_{T_{i}}=\left(\boldsymbol{R}_{i}-\boldsymbol{R}_{M}\right) \times \boldsymbol{b}_{F_{i}}, \quad \forall i \in \mathcal{S}_{\text {all }}
$$

where " $\times$ " denotes the cross product. $\boldsymbol{R}_{M} \in \mathbb{R}^{3}$ is the position vector of the Center of Mass (CoM), and $\boldsymbol{R}_{i} \in \mathbb{R}^{3}, \forall i \in \mathcal{S}_{\text {all }}$ are the position vectors of the thrusters, both expressed in the chaser body-fixed frame $\mathcal{F}_{b}$.

By analysing the configuration matrices $\boldsymbol{B}_{F}$ and $\boldsymbol{B}_{T}$ in terms of directional properties, the following can be concluded: thruster indices inside the sets $\mathcal{S}_{T i}, i=1, \ldots, 5$ have similar torque directions and are defined as

$$
\begin{array}{ll}
\mathcal{S}_{T 1}=\{1,11\}, & \mathcal{S}_{T 3}=\{4,8\}, \quad \mathcal{S}_{T 5}=\{3,6,9,12\} \\
\mathcal{S}_{T 2}=\{2,10\}, & \mathcal{S}_{T 4}=\{5,7\},
\end{array}
$$

In terms of force directions, the following is revealed

$$
\begin{array}{lll}
\boldsymbol{b}_{F_{1}}=-\boldsymbol{b}_{F_{11}}, & \boldsymbol{b}_{F_{4}}=-\boldsymbol{b}_{F_{8}}, & \boldsymbol{b}_{F_{3}}=-\boldsymbol{b}_{F_{12}} \\
\boldsymbol{b}_{F_{2}}=-\boldsymbol{b}_{F_{10}}, \quad \boldsymbol{b}_{F_{5}}=-\boldsymbol{b}_{F_{7}}, & \boldsymbol{b}_{F_{6}}=-\boldsymbol{b}_{F_{9}}
\end{array}
$$

which means that the thruster pairs given by $\mathcal{S}_{T i}, i=1, \ldots 4$ produce exactly opposite forces. The last thruster group, i.e. $\mathcal{S}_{T 5}$, has the following orthogonal property

$$
\boldsymbol{b}_{F_{3}} \cdot \boldsymbol{b}_{F_{6}}=0, \quad \boldsymbol{b}_{F_{9}} \cdot \boldsymbol{b}_{F_{12}}=0
$$

where ". "denotes the dot product.

Assuming no simultaneous faults, the considered thruster faults can be modeled in a multiplicative way according to:

$$
\boldsymbol{u}_{f}(t)=(\boldsymbol{I}-\boldsymbol{\Psi}(t)) \boldsymbol{u}(t), \quad \boldsymbol{\Psi}(t)=\operatorname{diag}\left(\psi_{1}(t) \ldots \psi_{12}(t)\right)
$$

where $0<\psi_{i} \leq 1, \forall i \in \mathcal{S}_{\text {all }}$ are unknown. $\psi_{i}$ is about to model closed fault types, i.e. $i^{t h}$ thruster blocked-closed $\left(\psi_{i}=\right.$ $1)$ and/or loss of efficiency $\left(0<\psi_{i}<1\right)$ of the $i^{t h}$ thruster. 


\section{A. Fault detection based on position model}

The proposed fault detector design is based on the relative position model of the chaser and target expressed in the local (target) reference frame $\mathcal{F}_{l}=\left\{O_{l} ; \hat{\boldsymbol{x}}_{l}, \hat{\boldsymbol{y}}_{l}, \hat{\boldsymbol{z}}_{l}\right\}$. The concerned reader can found further details on modeling the relative dynamics of two spacecrafts in the available space literature, see for instance [16]. A linear $6^{\text {th }}$ order state space model with state vector $\boldsymbol{x}=[x y z \dot{x} \dot{y} \dot{z}]^{T}$ modeling the chaser relative motion expressed in $\mathcal{F}_{l}$, both in fault free (i.e. $\Psi=0$ ) and faulty (i.e. $\boldsymbol{\Psi} \neq \mathbf{0}$ ) situations is given by

$$
\begin{aligned}
& \dot{\boldsymbol{x}}(t)=\boldsymbol{A}_{p} \boldsymbol{x}(t)+\boldsymbol{B}_{p} \boldsymbol{R}\left(\hat{\boldsymbol{q}}_{t}(t), \hat{\boldsymbol{q}}_{c}(t)\right) \boldsymbol{B}_{F} \boldsymbol{u}_{f}(t-\tau(t)) \\
& \boldsymbol{y}(t)=\boldsymbol{C}_{p} \boldsymbol{x}(t)
\end{aligned}
$$

where the rotation matrix $\boldsymbol{R}\left(\hat{\boldsymbol{q}}_{t}, \hat{\boldsymbol{q}}_{c}\right)$ is calculated from the quaternion estimates of the chaser $\hat{\boldsymbol{q}}_{c} \in \mathbb{R}^{4}$ and the target $\hat{\boldsymbol{q}}_{t} \in \mathbb{R}^{4}$ attitude, and rotates the force vector from $\mathcal{F}_{b}$ into $\mathcal{F}_{l}$. These estimates come from the navigation unit. The output vector $\boldsymbol{y}=\left[\begin{array}{ll}x y & z\end{array}\right]^{T}$ is the relative position in $\mathcal{F}_{l}$ measured by a Light Detection and Ranging (LIDAR) device.

In [7], a sensitivity/robustness analysis was performed showing high reliability and efficiency (in terms of detection times) of a fault detector based on a position model in $\mathcal{F}_{l}$. Here, an observer-based fault detector is designed that has enhanced robustness to above mentioned time delay $\tau(t)$. This observer uses the model given in (32) and (33) to generate the state estimate $\hat{\boldsymbol{x}}$ used to produce the residual $\boldsymbol{r}=\left[r_{1}, r_{2}, r_{3}\right]^{T}$, i.e.

$$
\boldsymbol{r}(t)=\boldsymbol{Q}\left(\boldsymbol{y}(t)-\boldsymbol{C}_{p} \hat{\boldsymbol{x}}(t)\right)
$$

where $Q$ is a weighting matrix. The design of (34) is based on theoretical developments given in [3], using the Padé method.

The proposed decision making rule is based on the scalar valued Generalized Likelihood Ratio (GLR) test given in [17]. The decision test $\varrho(t)$ is then defined by:

$$
\varrho(t)= \begin{cases}1 & S(k)>J_{t h} \Rightarrow \text { fault declared } \\ 0 & S(k) \leq J_{t h} \Rightarrow \text { fault not present }\end{cases}
$$

with $S(k)=\sum_{i=1}^{3} w_{i} S_{i}(k)$, where $w_{i} \geq 0, i=1,2,3$ are the normalized weight factors used to prioritize certain elements (axis) of the residual, $S_{i}(k)$ is the estimated likelihood of the GLR algorithm applied to the $i^{\text {th }}$ residual $r_{i}(k)$ evaluated at time instant $t=k T_{s}, k \in \mathbb{Z}^{+}$where $T_{s}$ is the navigation sampling time, and $J_{t h}$ is a fixed threshold.

\section{B. Thruster group isolation using a bank of NUIOs}

Recalling the thruster configuration properties given by (28)(30), we assume that it is easier to get explicit information from the angular velocity $\boldsymbol{\omega} \in \mathbb{R}^{3}$ measurement than from the linear position/velocity. Therefore, the model of the attitude dynamics of a rigid-body spacecraft, i.e.

$$
\boldsymbol{J} \dot{\boldsymbol{\omega}}(t)=\boldsymbol{B}_{T} \boldsymbol{u}_{f}(t)-\boldsymbol{\omega}(t) \times \boldsymbol{J} \boldsymbol{\omega}(t)
$$

is used for the design of a bank of NUIOs. In $(36), \boldsymbol{J} \in \mathbb{R}^{3 \times 3}$ stands for the inertia of the chaser in $\mathcal{F}_{b}$. The adjustable NUIO, introduced in section III, has been selected because of its decoupling properties, ability to take into account nonlinearities of the attitude dynamics (36) and adjustable error dynamics.

The attitude model (36) can be represented in the form of (6) and (7) with the following assignment: $x=\omega$, $\boldsymbol{f}(\boldsymbol{\omega})=-\boldsymbol{J}^{-1} \boldsymbol{\omega} \times \boldsymbol{J} \boldsymbol{\omega}, \boldsymbol{A}=\mathbf{0}, \boldsymbol{B}=\boldsymbol{J}^{-1} \boldsymbol{B}_{T}$, and $\boldsymbol{C}=\boldsymbol{I}$. One may argue that $\boldsymbol{f}(\boldsymbol{\omega})$ is not globally Lipschitz, because the Jacobian $\partial \boldsymbol{f} / \partial \boldsymbol{\omega}$ is not uniformly bounded over $\mathbb{R}^{3}$. However, $\boldsymbol{f}(\boldsymbol{\omega})$ is continuously differentiable on $\mathbb{R}^{3}$. Thus, it is locally Lipschitz. This means that the angular velocity shall be bounded in magnitude which is a reasonable assumption from a practical point of view. Using a constrained optimization algorithm, one can find a Lipschitz constant $\kappa$ over the set $\mathcal{S}=\left\{\boldsymbol{\omega} \in \mathbb{R}^{3}:\left|\omega_{i}\right| \leq \bar{\omega}, i=1,2,3\right\}$, where $\bar{\omega}$ is the upper bound of the angular velocity for each axis.

For each thruster group $\mathcal{S}_{T i}$, a dedicated NUIO is thus designed. Each NUIO is such that it can fully estimate the angular velocity with all the inputs except those belonging to $\mathcal{S}_{T i}$, i.e. $u_{i}, i \in \mathcal{S}_{a l l} \backslash \mathcal{S}_{T i}$. As a result, the NUIO dedicated to the group $\mathcal{S}_{T i}$ will not be affected by faults occurring in the thrusters belonging to $\mathcal{S}_{T i}$, while all the other NUIOs will be. The proposed method is summarised by Algorithm 1 .

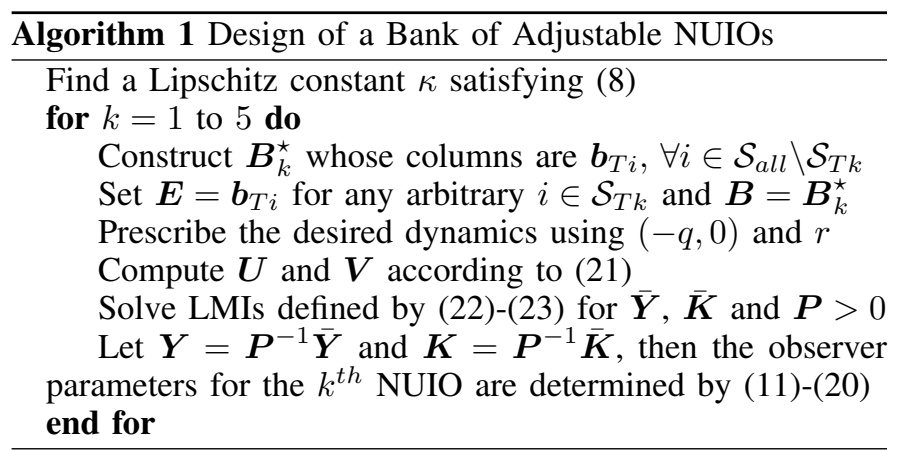

This suggests the following isolation procedure: defining the angular velocity estimation error of the $i^{t h}$ observer as $\boldsymbol{e}_{i}(t)=$ $\hat{\boldsymbol{\omega}}_{i}(t)-\boldsymbol{\omega}(t)$, then the faulty thruster group $\mathcal{S}_{T i}$ is identified based on the following rule

$$
\sigma_{g}(t)=\arg \min _{i \in \mathcal{G}_{T}}\left\|\boldsymbol{e}_{i}(t)\right\|, \quad t>t_{d}
$$

where $t_{d}$ is the fault detection time, i.e. the time when the fault is declared by $\varrho(t), \mathcal{G}_{T}=\{1,2, \ldots 5\}$ denote the set of all indices linked with the thruster groups $\mathcal{S}_{T 1}, \ldots, \mathcal{S}_{T 5}$, and the function $\sigma_{g}(t): \mathbb{R}^{+} \rightarrow \mathcal{G}_{T}$ represents the identified thruster group index that is most likely affected by a fault.

\section{Thruster isolation logic}

Once a thruster group $\mathcal{S}_{T i}$ is identified by $\sigma_{g}(t)$, the faulty thruster can be easily isolated by examining the angle of the vector $\boldsymbol{r}$ given by (34) along the thruster directions $\boldsymbol{d}_{i}, i \in \mathcal{S}_{T i}$. If the $i^{t h}$ thruster is faulty, then, the vectors $\boldsymbol{r}$ and $\boldsymbol{d}_{i}$ should be collinear (owing the fault model (31)). Using the directional cosine approach, the following isolation logic reveals

$$
\sigma(t)=\arg \max _{j \in \mathcal{S}_{T i}} \frac{\boldsymbol{d}_{j}^{T} \boldsymbol{r}(t)}{\left\|\boldsymbol{d}_{j}\right\|\|\boldsymbol{r}(t)\|}
$$

which results in the thruster index matching the faulty thruster. Only thrusters belonging to the (already) identified group $\mathcal{S}_{T i}$ are tested in (38). The thruster directions within the groups $\mathcal{S}_{T i}, i \in \mathcal{G}_{T}$ are either exactly opposite, see 29, or are orthogonal, see (30), what makes the isolation logic $\sigma(t): \mathbb{R}^{+} \times \mathcal{G}_{T} \rightarrow \mathcal{S}_{\text {all }}$ very reliable. The proposed FDI strategy is summarised by algorithm 2 .

To avoid initial transition phenomena and to ensure robustness, two confirmation windows are introduced in Algorithm 2 , i.e. $\delta_{g}>0$ for $\sigma_{g}(t)$ and $\delta>0$ for $\sigma(t)$. 


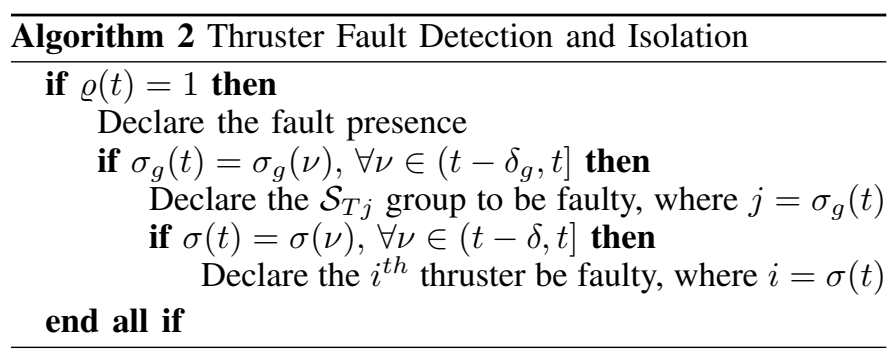

\section{FAUlT ACCOMMODATION}

The investigated thruster configuration disposes of an additional freedom to achieve fault tolerance, i.e. it is possible to achieve admissible GNC performance even if only $N-1$ (healthy) thrusters are used to control the spacecraft. The nominal controller is designed based on certain predetermined performance criteria. Since it is desirable to keep the nominal controller in the loop, the proposed solution consists in performing the fault accommodation using control re-allocation.

The proposed fault accommodation strategy works as follows: as soon as the faulty thruster index is clearly isolated by Algorithm 2, the faulty thruster is turned off using the dedicated thruster latch valve and the desired forces and torques are re-allocated among the $N-1$ healthy thrusters. Here, the quadratic programming approach is used. This problem is posed as the following Sequential Least-Squares (SLS) problem:

$$
\begin{gathered}
\boldsymbol{u}=\arg \min _{\boldsymbol{u} \in \mathcal{M}}\left\|\boldsymbol{W}_{u}\left(\boldsymbol{u}-\boldsymbol{u}_{d}\right)\right\| \\
\mathcal{M}=\arg \min _{\mathbf{0} \leq \boldsymbol{u} \leq \overline{\boldsymbol{u}}}\left\|\boldsymbol{W}_{v}\left(\boldsymbol{B}_{a} \boldsymbol{u}-\boldsymbol{v}_{d}\right)\right\|
\end{gathered}
$$

where $\boldsymbol{B}_{a}^{T}=\left[\begin{array}{ll}\boldsymbol{B}_{F}^{T} & \boldsymbol{B}_{T}^{T}\end{array}\right]$ is the overall configuration matrix, $\boldsymbol{v}_{d}$ is the vector of the desired forces and torques, and $\overline{\boldsymbol{u}}=\left[\bar{u}_{1}, \ldots, \bar{u}_{12}\right]^{T}$ are the upper limits defined as: $\bar{u}_{j}=$ $1, \forall j \in \mathcal{S}_{\text {all }} \backslash \sigma(t)$ and $\bar{u}_{i}=0, i=\sigma(t)$. This optimization problem should be interpreted as follows: given $\mathcal{M}$, the set of feasible control inputs that minimize $\boldsymbol{B}_{a} \boldsymbol{u}-\boldsymbol{v}_{d}$ (weighted by $\boldsymbol{W}_{v}$ ), pick the control input that minimizes $\boldsymbol{u}-\boldsymbol{u}_{d}$ (weighted by $\left.\boldsymbol{W}_{u}\right)$. Here, $\boldsymbol{u}_{d}$ is the desired control input and $\boldsymbol{W}_{u}$ and $\boldsymbol{W}_{v}$ are nonsingular weighting matrices. $\boldsymbol{W}_{u}$ affects the control distribution among the thrusters and $\boldsymbol{W}_{v}$ affects the prioritization among the virtual control components when $\boldsymbol{B}_{a} \boldsymbol{u}-\boldsymbol{v}_{d}$ cannot be attained due to, e.g. thruster constraints. A faster algorithm can be obtained by approximating the SLS formulation as a Weighted Least-Squares (WLS) problem:

$$
\begin{gathered}
\min \left\|\boldsymbol{W}_{u}\left(\boldsymbol{u}-\boldsymbol{u}_{d}\right)\right\|^{2}+\gamma\left\|\boldsymbol{W}_{v}\left(\boldsymbol{B}_{a} \boldsymbol{u}-\boldsymbol{v}_{d}\right)\right\|^{2} \\
\text { subj.to } \mathbf{0} \leq \boldsymbol{u} \leq \overline{\boldsymbol{u}}
\end{gathered}
$$

As $\gamma \rightarrow \infty$, the two formulations have the same optimal solution $\boldsymbol{u}$. An iterative Fixed-Point (FXP) algorithm can be used to solve the WLS formulation 441, see [18] for implementation details. This algorithm asymptotically converges to the optimal solution and the maximum number of iteration $N_{c a}$ can be considered to reflect the maximum computation time available.

\section{Simulations}

The thruster Fault Detection, Isolation and Accommodation (FDI-A) strategy described in the previous sections is implemented within the MSR "high-fidelity" industrial simulator provided by Thales Alenia Space. Following the design steps given in Algorithm 1, a bank of 5 adjustable NUIOs is designed

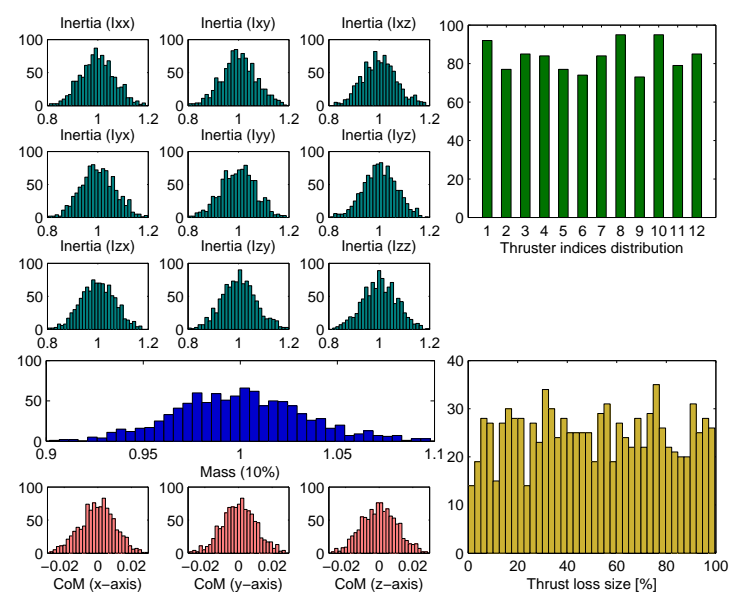

Fig. 1. Inertia (top left), mass (middle left), CoM (bottom left), thruster indices (top right), and thrust loss size (bottom right) distribution, respectively

with $\kappa=0.2, q=0.5$ and $r=0.1$. The FXP algorithm is used for control re-allocation with $\boldsymbol{W}_{v}=\boldsymbol{I}, \boldsymbol{W}_{u}=\boldsymbol{I}, \boldsymbol{u}_{d}=\mathbf{0}$, $N_{c a}=200$, and $\gamma=10^{6}$. The remaining design parameters are chosen as follows: $\boldsymbol{Q}=\boldsymbol{I}, J_{t h}=300, T_{s}=0.1 \mathrm{~s}$, $\delta_{g}=1 s, \delta=1 s$, and $w_{i}=1 / 3, \forall i \in\{1,2,3\}$. A set of 1000 Monte Carlo (MC) simulations is performed in order to assess the performance and robustness of the proposed FDI-A scheme. The navigation is considered to deliver "non-perfect" estimates. We also assume delays induced by the CPDE unit, $1 \%$ uncertainty on the thruster rise times, $\pm 3 \mathrm{~cm}$ uncertainty on CoM (thus uncertain $\boldsymbol{B}_{T}$ ), $10 \%$ uncertainty on mass, $20 \%$ uncertainty on inertia, and spatial disturbances (i.e. gravity gradient, atmospheric drag, and solar radiation pressure). See Fig. 1 for an illustration of considered uncertainties. All simulations are carried out during the last $20 \mathrm{~m}$ of the rendezvous phase (capture phase) and are associated with a fault scenario when the $i^{t h}$ thruster lose its effectiveness (thrust loss) of a size $\psi_{i} \in[0 \%, 100 \%]$. The other thrusters are fault-free. Faults starts at $t_{f}=1000 \mathrm{~s}$ and are maintained. The two marginal cases, i.e. $\psi_{i}=0 \%$ and $\psi_{i}=100 \%$, represent a nominal operational and fully closed thruster, respectively.

The effect of small thrust losses $\left(\psi_{i} \lesssim 15 \%\right)$ is relatively small on the system dynamics and shall be compensated by a robust control law. On the other hand, these faults are very hard or even impossible to detect and isolate. The aim of this MC simulation campaign is to show that if the FDI unit fails to detect or isolate the faulty thruster, the effect that this fault has on the GNC system and/or on the final MSR capture performance requirements is negligible. As seen in Fig. 2, despite the fact that in some cases the FDI unit failed, the final capture requirements in terms of position and velocities are fully met. The final attitude and angular rate error requirements (see Fig. 3) are met in $97.9 \%$ and $96.2 \%$ simulation cases, respectively. These results may be further improved by fine-tuning the FDI scheme (e.g. by adjusting the NUIOs dynamics). Note that, in some cases, the angular rate error requirement is not met even if the FDI succeed. This can be the case when it took too long for the FDI unit to detect and/or isolate the faulty thruster, thus the fault accommodation unit has not enough time to fully recover the faulty system. Figure 4 illustrates that the chaser maintains the nominal trajectory, i.e. stays inside the rendezvous corridor, and that the chaser keeps its attitude pointing towards the target, thus the target remains visible from the sensors. 


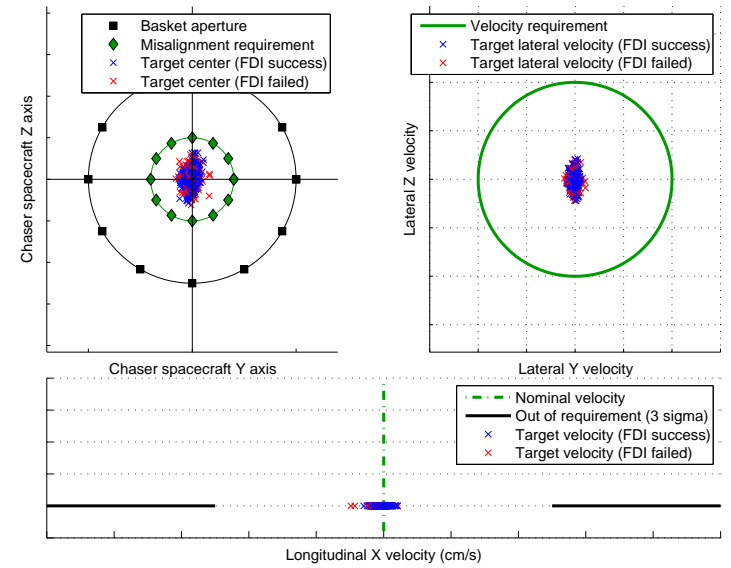

Fig. 2. MSR capture performance: position misalignment on $+\mathrm{X}$ face (top left), lateral velocity (top right) and longitudinal velocity (bottom) errors

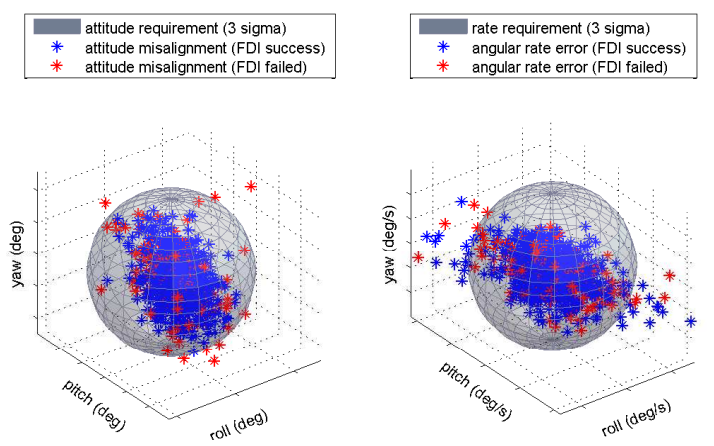

Fig. 3. Final attitude misalignments (left) and final angular rate errors (right)

\section{CONCLUSION}

A method to detect, isolate, and accommodate thruster faults of an autonomous spacecraft has been studied in this paper. The FDI unit consists of a bank of nonlinear unknown input observers with adjustable dynamics, a fault detector that is based on judiciously chosen position model and an isolation logic that uses the directional cosine approach. Once a fault is isolated, a control re-allocation technique redistributes the desired force and torque vectors among the remaining $N-1$ healthy actuators. This makes the FDI-A without any change in the nominal controller, without any redundant thruster set or without any additional valve position sensor. Results from the MC simulation campaign show that the proposed FDI-A scheme is able to accommodate thruster faults that may have effect on the GNC performance and on the rendezvous criteria.

\section{ACKNOWLEDGMENT}

This research work was supported by ESA and Thales Alenia Space in frame of ESA's Networking/Partnering Initiative.

\section{REFERENCES}

[1] X. Olive, "FDI(R) for satellites: How to deal with high availability and robustness in the space domain?" International Journal of Applied Mathematics and Computer Science, vol. 22, no. 1, pp. 99-107, 2012.

[2] A. Zolghadri, D. Henry, J. Cieslak, D. Efimov, and P. Goupil, Fault Diagnosis and Fault-Tolerant Control and Guidance for Aerospace Vehicles, ser. Advances in Industrial Control. Eds. Springer, 2014.

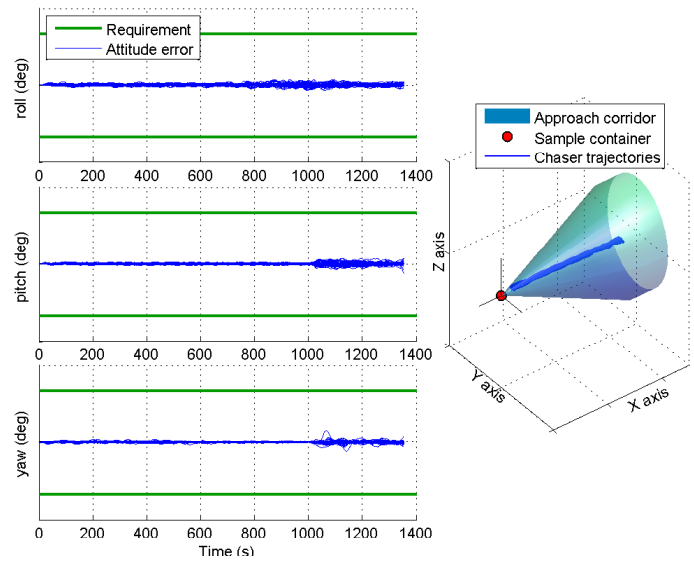

Fig. 4. LIDAR sensor field of view requirement (left) and the MSR rendezvous corridor (right)

[3] R. Fonod, D. Henry, E. Bornschlegl, and C. Charbonnel, "Robust fault diagnosis for systems with electronic induced delays," in Proc. of Workshop on Advanced Control and Diagnosis, Copenhagen, DK, 2012.

[4] W. Chen and M. Saif, "Observer-based fault diagnosis of satellite systems subject to time-varying thruster faults," Journal of Dynamic Systems, Measurement and Control, vol. 129, no. 3, pp. 352-356, 2007.

[5] D. Henry, "Fault diagnosis of microscope satellite thrusters using $H_{\infty} / H_{-}$filters," Journal of Guidance, Control, and Dynamics, vol. 31, no. 3, pp. 699-711, 2008.

[6] A. Falcoz, F. Boquet, M. Dinh, B. Polle, G. Flandin, and E. Bornschlegl, "Robust fault diagnosis strategies for spacecraft application to LISA pathfinder experiment," in Proc. of IFAC Symposium on Automatic Control in Aerospace, Nara, Japan, 2010, pp. 404-409.

[7] R. Fonod, D. Henry, E. Bornschlegl, and C. Charbonnel, "Robust fault detection for systems with electronic induced delays: Application to the rendezvous phase of the MSR mission," in Proc. of European Control Conference, Zurich, Switzerland, 2013, pp. 1439-1444.

[8] Y. Zhang and J. Jiang, "Bibliographical review on reconfigurable faulttolerant control systems," Annual Reviews in Control, vol. 32, no. 2, pp. 229-252, 2008.

[9] M. Hou and P. Müller, "Design of observers for linear systems with unknown inputs," IEEE Transactions on Automatic Control, vol. 37, no. 6, pp. 871-875, 1992.

[10] M. Darouach, M. Zasadzinski, and S. Xu, "Full-order observers for linear systems with unknown inputs," IEEE Transactions on Automatic Control, vol. 39, no. 3, pp. 606-609, 1994.

[11] A. Filasova and D. Krokavec, "On the Takagi-Sugeno model-based state estimation for one class of bilinear systems," in Prof. of 14th International Carpathian Control Conference, 2013, pp. 83-87.

[12] A. Pertew, H. Marquez, and Q. Zhao, "Design of unknown input observers for Lipschitz nonlinear systems," in Proc. of American Control Conference. Portland, OR, USA: IEEE, 2005, pp. 4198-4203.

[13] W. Chen and M. Saif, "Unknown input observer design for a class of nonlinear systems: an LMI approach," in Proc. of American Control Conference. Minneapolis, Minnesota USA: IEEE, 2006, pp. 834-838.

[14] R. Fonod, D. Henry, E. Bornschlegl, and C. Charbonnel, "Thruster fault detection, isolation and accommodation for an autonomous spacecraft," in Proc. of IFAC World Congress, Cape Town, South Africa, 2014.

[15] M. Chilali and P. Gahinet, " $H_{\infty}$ design with pole placement constraints: An LMI aproach," IEEE Transactions on Automatic Control, vol. 41, no. 3, pp. 358-367, 1996.

[16] H. Schaub and J. Junkins, Analytical Mechanics of Space Systems. Reston, VA: AIAA Education Series, 2009.

[17] S. X. Ding, Model-based Fault Diagnosis Techniques: Design Schemes, Algorithms, and Tools, 1st ed. Springer Verlag, 2008.

[18] J. J. Burken, P. Lu, Z. Wu, and C. Bahm, "Two Reconfigurable Flight-Control Design Methods: Robust Servomechanism and Control Allocation," Journal of Guidance, Control, and Dynamics, vol. 24, no. 3, pp. 482-493, May 2001. 